\title{
INTERFERENCE OF CELLULAR PHONE CONVERSATIONS WITH VISUOMOTOR TASKS: AN ERP STUDY
}

\author{
Authors: Luis García-Larrea, Caroline Perchet, Fabien Perrin and Elena Amenedo
}

This is the peer reviewed version of the following article: García-Larrea, L., Perchet, C., Perrin, F., Amenedo, E. (2001). Interference of cellular phone conversations with visuomotor tasks: An ERP study. Journal of Psychophysiology, 15, 14-21. doi: 10.1027//0269-8803.15.1.14.

This article may be used for non-commercial purposes in accordance with Hogrefe and Federation of European Psychophysiology Societies terms and conditions for use of self-archived versions. 


\title{
Interference of Cellular Phone Conversations with Visuomotor Tasks: An ERP Study
}

\author{
Authors: Luis García-Larrea ${ }^{1,2}$, Caroline Perchet ${ }^{1}$, Fabien Perrin ${ }^{1}$, and Elena Amenedo ${ }^{3}$ \\ ${ }^{1}$ Research Unit (UPRES) EA 1880, Université Claude Bernard, Lyon, France \\ ${ }^{2}$ Human Neurophysiology Laboratory at the CERMEP, INSERM, Lyon, France \\ ${ }^{3}$ Department of Clinical Psychology and Psychobiology, University of Santiago de Compostela, Galicia, \\ Spain
}

Corresponding autor: Luis García-Larrea via email at larrea@univ-lyon1.fr

\section{Post-print (final draft post-refereeing)}




\begin{abstract}
The use of mobile phones has been shown to increase drivers' reaction times (RTs), but whether this results from interference with attention, stimulus identification, or response production remains unclear. We recorded RTs and event-related brain potentials (ERPs) reflecting speed of stimulus processing, attentional allocation, and preparedness to respond during a visual reaction task performed with or without the concomitant use of a mobile phone, in either "hands-free" or "phone-in-hand" operating modes. As expected, maintaining a phone conversation increased RTs to visual targets, this effect being associated with complex ERP effects. Phone conversations did not appear to delay target detection times, as assessed by N2-P3 latencies, but did significantly decrease stimulus-induced alerting and attentional allocation (P3 amplitude) and interfered with motor preparation processes (readiness potential). P3 amplitude drop was identical whatever the mode of phone use, while decrease of readiness potential was progressive from the "hands-free" to the "phone-in-hand" condition. These results suggest that two mechanisms contributed to degrade performance in this experiment: first, a general decrease of attention to sensory inputs, characteristic of "dual-task" situations, probably acting through a delay in sensory-motor transfer times. This effect was independent of whether the phone was handled or "hands-free." Conversely, the second factor was specifically sensitive to manipulation of the phone and caused a weakening of the readiness to respond with a motor act.
\end{abstract}

\title{
Post-print (final draft post-refereeing)
}




\section{Introduction}

Mobile cellular telephones are being increasingly used in developed countries, and a growing proportion of phone conversations are conducted during car driving. Several behavioral investigations have addressed the question of the effects that mobile telephone use may have on traffic safety, as it appears unlikely that a complex situation like car driving may be insensitive to the superimposed demands of a concurrent phone conversation. Before the dissemination of car telephones, it was suggested that phone usemight led to decrements in both perception and decision making during driving (Brown et al., 1969), and other authors later observed negative effects of phone use on driving performance, including lateral path deviations (Briem \& Hedman, 1995), increased heart rate and subjective sensation of effort (Brookhuis et al., 1991), and delayed reaction times to rare events (Alm \& Nilsson, 1995). Using road position, driving speed, and collisions as dependent variables, Briem and Hedman (1995) found that the handling of the telephone and the maintenance of a difficult conversation had adverse effects on performance, while "easy talking" over a hands-free telephone could, in some cases, be seen as facilitatory. Along this line, Preece et al. (1999) and Koivisto et al. (2000) recently reported a possible facilitation of motor responsiveness (shorter reaction times) in subjects receiving microwave radiation at $902-915 \mathrm{MHz}$, similar to that emitted by cellular phones.

Behavioral studies have introduced important data on performance decrements derived from the use of mobile phones, but have not explored the mechanisms underlying such anomalies. This is an important issue, as almost identical alterations in overt behavior may result from a variety of processing deficits. For instance, increased reaction times (RTs) to environmental targets during phone conversations (Alm \& Nilsson, 1995) may result either from a deficit in stimulus evaluation, a lack of anticipation, preparation and execution of motor responses, or any combination of these deficits.

Event-related brain potentials (ERPs) show variations of electrical brain activity associated with the processes of attending to, anticipating, and detecting environmental targets, as well as reflecting the preparation to make a motor response. Compared to behavioral measures, one theoretical advantage of ERP analyses is therefore the study of processes that are hardly accessible to RT measurements. The application of these techniques in combination with behavioral data may help understand the respective contributions of attentional, perceptual and anticipation/preparation processes to the observed behavioral deficits. We therefore used ERPs to investigate the mechanisms underlying changes in RTs to sensory targets during the use of mobile phones. To this end, brain responses were recorded during a simple-reaction, visual-detection task of targets versus distractors, performed concomitantly to a mobile phone conversation.

We investigated whether or not the concomitant use of a telephone entailed (a) a delay in stimulus identification; (b) a reduction in attention allotted to the task and stimulus- related arousal; and (c) a decrease in preparatory processes. Stimulus detection times were estimated using the timing of the N2-P3 complex in poststimulus ERPs, which is a common measure of stimulus evaluation time (Kutas et al., 1977; Donchin, 1979; Picton, 1992). The amplitude of the P3 wave covaries with the amount of attentional resources allotted to a target stimulus (Wickens et al., 1984; Kok, 1997; García-Larrea et al., 1998), and with stimulus-related arousal (Bahramali et al., 1997; Kok, 1997), and was used accordingly to assess these variables. Finally, slow negative potentials preceding the target stimulus were also recorded. Such slow potentials correspond to

\section{Post-print (final draft post-refereeing)}


anticipation/orienting processes (CNV; Walter et al., 1964), to motor preparation (readiness potential or RP; Kornhuber et al., 1965) or to a combination of the two. In this study we used rapid sequences of target and nontarget stimuli requiring simple motor reactions; in these conditions there is evidence that the major portion of the prestimulus negativity consists of a readiness potential (see Barrett et al., 1987), which disappears if a motor response is replaced by a mental count (Starr et al., 1995). This potential, probably sharing properties with the "late" component of the contingent negative variation (McCallum, 1988), served as a marker of prestimulus activation and preparation processes to respond with a motor act.

In order to dissociate the specific effect of manipulating the telephone from the effects due to the conversation itself, both "hands-free" and "phone-in-hand" conditions were used in the experiments. Also, to avoid contamination by possible spurious effects in the EEG or ERPs linked to the electromagnetic radiation (Eulitz et al., 1998; Freude et al., 2000), all recordings were conducted with telephones switched off.

\section{Subjects \& Methods}

\section{Participants}

Ten healthy subjects ( 7 women, 3 men) aged 24 to 42 years $(32.6 \pm 7.2)$ participated in the study. Participants were free of neurological impairment, and had normal or corrected-to-normal vision and hearing. Each of them gave informed consent to participate in the experimental sessions. Two visual stimuli consisting respectively of a yellow sun (target) and a red circle (distractor), each subtending 1 degree of angle, were presented on the center of a computer screen against a black background, in random sequences. The duration of each stimulus was $200 \mathrm{~ms}$ and the interstimulus interval (ISI) was $1200 \mathrm{~ms}$. Red circles appeared with a probability of 0.8 and were randomly replaced by the yellow sun (target stimulus) with a probability of 0.2 . Subjects had to respond to the appearance of the target stimulus by pressing a mouse button with the dominant hand. Speed and accuracy were equally emphasized before each recording run. Atotal of 100 to 120 stimuli were presented in each trial.

\section{Experimental Procedure}

The task described above was presented to each subject in three experimental conditions. In condition 1 ("nophone") target detection was performed without any concomitant task. In condition 2 ("hands-free") the subjects maintained a "hands-free" mobile phone conversation while performing the target detection task; in this condition they could speak freely and hear through a loudspeaker the person they were speaking to. In condition 3 ("phone-in-hand"), subjects kept a phone conversation by holding a mobile phone in their nondominant hand while executing the reaction task. Two different standardized conversations were used for every subject. One was a business conversation including questions about job, studies, and hobbies, and the other consisted of questions about the experimental situation itself. Thus, to answer the questions in the first conversation the subject needed to access items relatively stabilized in long-term memory, the access to which was considered to be easy and almost automatic (e. g., favorite hobbies). The second conversation incorporated questions relevant to the ongoing experiment (type of setting, characteristics of stimuli, etc.), the response to which could not rely on previous knowledge and resulted in slightly higher subjective difficulty. In order to standardize the experimental situations, both conversations were previously recorded on tape and presented through an inserted earphone ("phone-in-hand" condition), or an external loudspeaker ("hands-

\section{Post-print (final draft post-refereeing)}


free" condition). Thus, every subject perceived the voice as if it came through the mobile phone, and each heard exactly the same voice and answered to the same questions as the others. This procedure also allowed us to discard any possible electromagnetic contamination from an active cellular phone, which has been shown to induce changes in both EEG and cognitive-evoked potentials (Eulitz et al., 1998). The order of conditions was counterbalanced across subjects, and the two conversations were also counterbalanced between the phone conditions. Subjects started the target detection task 5 to 10 seconds before receiving the phone call. In the "phone-in-hand" condition, they were instructed to take the phone in their nondominant hand, put it to the ear, and follow the conversation. In the "handsfree" condition, they had to push a button situated beside the phone, which automatically started the loudspeaker conversation.

\section{Data Recording and Analysis}

Motor reaction times (RTs) to target stimuli, as well as number of errors (omissions and false alarms) were recorded for each subject and condition. The electroencephalogram (EEG) was recorded continuously from tin electrodes $(\mathrm{Fz}, \mathrm{Cz}, \mathrm{Pz})$ mounted on an elastic helmet (ElectroCap ${ }^{\circledR}$ ) and referenced to the nose. The EEG was amplified 20,000 times with a bandpass of $0.1-30 \mathrm{~Hz}(-3 \mathrm{~dB})$ and sampled at $500 \mathrm{~Hz}$ with a Synamps ${ }^{\circledR}$ acquisition system. The electrooculogram (EOG) was also monitored by a tin electrode attached to the supero-lateral margin of the left superciliary arch, referenced to the nose. An electrode placed between Fz and Fpz served as ground. Skin impedances were kept below $5 \mathrm{~K} \Omega$. The recording settings of the experiments were based on those described by Starr et al. (1995). The raw EEG trace was segmented into epochs of 2 seconds centered on stimulus presentation, thus allowing simultaneously recording of ERPs preceding and following the stimulus, including prestimulus slow negativities (RP) and poststimulus N2 and P3 responses (Starr et al., 1995). Segmented epochs were then averaged for each subject and condition according to the type of stimulus (target or distractor). Prior to averaging, EEG epochs were inspected visually to detect and eliminate any segment contaminated by EOG artifacts. After EOG correction, single epochs with amplitudes exceeding $\pm 70 \mu \mathrm{V}$ were excluded from the average. Only ERPs to target stimuli are presented in this report.

The following were analyzed for each electrode:

- the peak latencies of the N2 \& P3 components;

- the P3 offset latency (point where the P3 ascending (negative-going) limb crossed the baseline);

- the P3 amplitude to baseline;

- the area under the slow negative shift preceding the stimulus.

For all latency measures the reference point was the onset of the target stimulus. The area under the prestimulus negativity was measured within an interval starting $600 \mathrm{~ms}$ prestimulus and extending until $50 \mathrm{~ms}$ poststimulus.

The amplitude of poststimulus ERPs was measured after applying a high-pass digital filter with cutoff at $1 \mathrm{~Hz}(6 \mathrm{~dB}$ down, $-24 \mathrm{~dB} / \mathrm{oct})$ to eliminate the baseline drift due to slow prestimulus potentials (Starr et al., 1995); then, ERP amplitudes were measured relative to a $400 \mathrm{~ms}$ prestimulus baseline (Figure 1, right panel). Latency windows for N2 and P3 analyses were determined from the grand-averaged waveforms across all subjects in the control condition, and were $200-300 \mathrm{~ms}$ and 300-500ms poststimulus respectively for N2 and P3. In case of multiple peaks on one component, latency was calculated as the intersection between the ascending and

\section{Post-print (final draft post-refereeing)}


descending branches (Goodin et al., 1994). The "offset latency" of the P3 component was calculated as the point at which the ascending (negative-going) branches of the potential intersected with the baseline, after applying the high-pass filter described above. Behavioral and ERP latency data were subjected to repeated measures ANOVAs, with condition (3 levels) and electrode (3 levels) as within-subject factors for ERP data, and condition only for behavioral data. The Greenhouse- Geisser $(\mathrm{G}-\mathrm{G}) \varepsilon$ estimate was used to correct the degrees of freedom when appropriate. Significant factor effects $(P=0.05$ after $\mathrm{G}-\mathrm{G}$ correction) were followed by post-hoc Student paired t-tests.

\section{Results}

\section{Behavioral Data}

Reaction times to targets increased from $348 \pm 80 \mathrm{~ms}$ in the control ("no-phone") situation, to 409 $\pm 110 \mathrm{~ms}$ in "hands-free" mobile use, and $418 \pm 110 \mathrm{~ms}$ in the "phone-in-hand" situation, the difference being highly significant $(\mathrm{F}(2,9)=12.79, \varepsilon=.9, \mathrm{P}<.001)$. Motor reactions were slower in both phone conditions than in the control situation (paired $t(9)$, respectively $-4.48, P=0.001$ and $-3.98, \mathrm{P}=0.003$ ), but the difference between the two phone conditions ( $409 \mathrm{~ms}$ vs $418 \mathrm{~ms}$ ) did not reach significance. False alarms and omissions were less than $1 \%$ in all conditions and did not differed among them.

\section{Electrophysiological Data}

The experimental condition had a significant effect on the slow negativity which preceded stimulus presentation $(\mathrm{F}(2,9)=4.01, \varepsilon=0.93, \mathrm{P}<.04)$, the mean area of which progressively decreased from maximal values in the "no-phone" situation, to intermediate in the "handsfree" condition, and minimal during the "phone-inhand" context, where the prestimulus negativity was virtually absent (Figure 1, left panel). Attenuation of prestimulus

negativity did not reach significance in the "hands-free" condition relative to the control experiment $(\mathrm{t}(9)=-1.78$, n.s.) but became highly significant when the phone was held in the hand $(\mathrm{t}(9)=-3.13, \mathrm{P}<.01)$.

The amplitude of the P3 component to targets was also significantly modified by phone use $(\mathrm{F}(2,9)=11.09, \varepsilon=.79, \mathrm{P}=.003)$. Amplitude decreased from $17.1 \pm 5 \mu \mathrm{V}$ in the "no-phone" condition to $10.2 \pm 5.2 \mu \mathrm{V}$ in the "handsfree" situation, and then remained relatively stable (9.1 $\pm 3.4 \mu \mathrm{V}$ ) with the phone-in-hand (Figure 1, right panel). Post-hoc t-tests disclosed significant amplitude decrease in both phone situations, relative to the control condition (respectively $t(9)=$ $3.36, \mathrm{P}=.008$ and $\mathrm{t}(9)=4.01, \mathrm{P}=.003)$, with no significant differences between "handsfree" and "phone-in-hand" ( $\mathrm{t}(9)=-1.03, \mathrm{P}=.33 \mathrm{n} . \mathrm{s})$. Amplitudes of $\mathrm{N} 2$ followed the same trend as those of $\mathrm{P} 3$, with amplitude decrease from the "no-phone" condition to both the "hands-free" and "phone-in-hand" situations (Figure 1, right panel), but the differences did not reach significance.

Peak latencies of the $\mathrm{N} 2$ and $\mathrm{P} 3$ components did not vary significantly among the three conditions (respectively $\mathrm{F}(2,9)=1.79$, n.s., and $\mathrm{F}(2,9)=.54$, n.s.). A significant effect of condition was observed on the $\mathrm{P} 3$ offset latency $(\mathrm{F}(2,9)=10.3, \varepsilon=.99, \mathrm{P}<.001)$, which increased during the two phone situations as compared with the control condition $(\mathrm{P}<.05)$, indicating longer $\mathrm{P} 3$ duration during phone use. Again, no difference was observed between the two phone conditions.

\section{Discussion}

\section{Post-print (final draft post-refereeing)}


Motor reaction times to target stimuli are a complex function of a number of variables, including at least:

a) anticipation and motor preparation processes, which accelerate reaction times by allowing faster accessibility to motor programs (Brunia, 1993);

b) stimulus-induced arousal, which increases response speed probably by acting on feature extraction \& response selection stages (Hackley \& Valle-Inclán, 1998);

c) stimulus evaluation speed, since response programs cannot be launched before the stimulus has been minimally evaluated.

Data from our study suggest that the use of a mobile phone concomitant with a visuo-motor task affected significantly at least two of these three variables. Electrophysiological recordings showed two main effects: attenuation of the slow negative shift preceding the stimulus, and attenuation of the N2-P3 response following target presentation. These two effects likely contributed to the degraded performances (delayed RTs) that were also observed, but had different dynamics (Figure 2) and were presumed to act through different mechanisms.

Slow negative potentials preceding an imperative, motor-demanding stimulus may correspond to anticipation/ orienting processes (CNV), to motor preparation (RP) or to a combination of these. The paradigm used in this study used relatively rapid sequences of target and nontarget stimuli requiring a simple motor reaction in response to the former. Barrett et al. (1987) observed that under these conditions the $\mathrm{N} 2$ and $\mathrm{P} 3$ components were more negative in the case of an imperative motor response than during mental counting, and inferred that a readiness potential was probably recorded in such target detection tasks.More recently, Starr et al. (1995) presented sequences of auditory stimuli requiring a simple target detection (20\% probability), and provided evidence that the major portion of the prestimulus negativity disappeared when the motor response was replaced by a mental count. They concluded, therefore, that, under these conditions, the prestimulus negativity mainly consisted of a readiness potential (RP) reflecting preparations to make a motor response. This potential, as with that recorded in this study, started about $500 \mathrm{~ms}$ before stimulus presentation, and probably shares properties with the "late" component of the contingent negative variation (McCallum, 1988). Attenuation of this RP in conditions 2 and 3 of our study suggests that motor preparedness to respond was significantly degraded by the concomitant use of the phone. Furthermore, the decrease of motor readiness potentials was different in the two phone conditions: only moderate in the "hands-free" situation and much more important when the phone had to be handled and manipulated - a situation where virtually no RP could be identified, as illustrated by the left panel of Figure 1. This extreme sensitivity of motor readiness potentials to the manipulation of the phone suggests that "hands-free" systems may be useful to preserve, at least in part, motor control mechanisms during phone conversations while executing other operations.

The second effect of mobile phone use was a decrease of P3 amplitude to visual targets. This is a classically observed effect of "dual-task" experiments, where the P3 evoked by one class of stimuli decreases when the load of the other task increases (Isreal et al., 1980; Wickens et al., 1984; Ragazzoni et al., 1996; Schubert et al., 1998; García-Larrea et al., 1998). Dual-task paradigms have stressed the value of P3 amplitude as an index of the capacity to process taskrelevant stimuli (Wickens et al., 1984; Picton, 1992; Kok, 1997). Since P3 amplitude is also positively related to phasic, stimulus-induced arousal (Bahramali et al., 1997; Bastuji \& García-

\section{Post-print (final draft post-refereeing)}


Larrea, 1999), P3 amplitude decrease in dual-task contexts may be viewed as the reflection of a decrease in target-induced arousal when attention is distributed among several sources of demands. Our results support the hypothesis that both attentional allocation to sensory stimuli, and phasic arousal to them were decreased during phone use. In addition, the fact that a similar P3 decrease was obtained whether the phone was held in the hand or not indicates that the main factor responsible for this effect was the conversation itself, rather than the manipulation of the phone. Thus, a decrease of the capacity to process external events appears as a direct consequence of the attentional load imposed by the phone conversation, whatever the mode of phone use, and is not likely to be ameliorated by the use of "hands-free" systems. Similar conclusions were reached in a recent field study, where reaction times to braking and/or collision were affected to a similar extent by a task involving phone manipulation and by a nonvisual attentional task, both performed concurrently to driving (Lamble et al., 1999).

In contrast to the above results, the time needed for identification and controlled classification of the visual targets, as indexed by N2 and P3 latencies, was not significantly altered by the concomitant use of the phone (although delay of P3 offset suggested increased variability in target processing during phone use). While $\mathrm{P} 3$, and especially $\mathrm{N} 2$, latencies represent a measure of stimulus processing time (Kutas et al., 1977; Ritter et al., 1979; Picton, 1992), RTs provide a complex estimate of both stimulus and response processing, including sensorimotor transfer (Donchin, 1979; Hackley \& Valle-Inclán, 1998). Our results suggest that delayed RTs while using the phone, although linked to decreased attention, were not principally due to a delay in stimulus classification and identification of the target. Divided attention settings may prolong RTs by affecting the cognitive-tomotor transfer (i. e., the stage whereby the output of sensory discrimination is used to launch a motor program) without necessarily delaying target evaluation time. Interestingly, conservation of target detection times appears to be common in dual-task situations, where a dissociation between preserved N2 and P3 latencies, yet delayed RTs, has often been documented (e. g., Sirevaag et al., 1989; Ragazzoni et al., 1996; García-Larrea \& Cézanne-Bert, 1998; Schubert et al., 1998). This and our present results suggest that stimulus evaluation times might be more resistant to dual-task contamination than cognitive-motor transfer times - at least in situations where stimulus detection is very simple. Stimulus detection delays might however become affected in the case of more complex attentional demands from one or both concurrent tasks, and this is being currently investigated in our laboratory.

The P3 "offset latency" was delayed during the two phone conditions (Figure 1). This did not appear to reflect a desynchronization ("jitter") of the P3 wave across trials, since only the ascending (negative going) slope of the late positive complex (LPC) was responsible for this effect, whereas the descending P3 branch was not affected.

Offset latency delay was therefore due to dissociation of late subcomponents of the LPC from the main P3 body, in a very similar way to that described in the context of other dual-task paradigms (García-Larrea \& Cézanne-Bert, 1998). Such late components have often been considered to reflect response selection stages (Falkenstein et al., 1994; Perchet \& García-Larrea, 2000).

We do not have enough evidence to attribute a definite functional value to these late components in the present study; however, in the context of theories that consider such late responses as response-related activities (Verleger, 1997)wemight assume that their delay may reflect a dissociation between stimulus-related processing (N2 and the first part of P3) and responseoriented (premotor) activities. Within this framework, the delay of P3 offset would further support

\section{Post-print (final draft post-refereeing)}


the hypothesis suggesting selective alteration of the "cognitive-to-motor" transfer by the concomitant use of the phone.

Interestingly, at least two recent reports have suggested a possible "facilitatory" effect of mobile phone use on reaction times and responsiveness to targets (Preece et al., 1999; Koivisto et al., 2000). These possible beneficial effects of mobile phones on cognition, which stand in disagreement with the present data and with previous behavioral reports (Alm \& Nilsson, 1995; Briem \& Hedman, 1995; Lamble et al., 1999), have been described while subjects received phonelike $(902-915 \mathrm{MHz})$ radiation to the lateral aspect of the head. More important, these experiments were conducted in the absence of phone manipulation and without the need to maintain any conversation through the phone, thus eliminating the most important factors of interference, i. e., the "divided- attention" setting and the manual task. The possible "beneficial" effect of electromagnetic radiation from a real cellular phone (manipulated and used to keep a conversation) in a dual-task context like driving has not, to our knowledge, been demonstrated. Possible interactions between electromagnetic radiation and "dual-task" effects are clearly beyond the scope of the present study, which on the contrary underscores the fact that both attentional and preparatory mechanisms in sensorimotor reaction tasks are negatively affected by concurrent mobile phone use.

\section{Conclusion}

We propose that at least two psychophysiological mechanisms may contribute to degrade performance when simple reaction tasks are executed concomitantly to cellular phone conversations. One is a general decrease of attention to sensory inputs, which has as a likely corollary a decrease in stimulus-induced arousal. This effect is characteristic of "dual-task" situations, appears related to the cognitive burden of adding a conversation to the reaction task, and is largely independent of the modality of phone use (hands-free or phone-in-hand). The second factor is a weakening of preparedness to respond with a motor act. Unlike the first factor, this effect was specifically sensitive to manipulation of the phone.

\section{Acknowledgments}

This work was supported by the "MAIF Foundation for Acident Research" ("Fondation MAIF de Recherche sur les Risques Accidentels," and benefited from discussions with Dr. G. Pachiaudi and A. Chapon, researchers at the INRETS (Institut National sur la Recherche sur les Transports et leur Sécurité).

\section{References}

Alm, H., \& Nilsson, L. (1995). The effects of a mobile telephone task on driver behavior in a car following situation. Accident Analysis and Prevention, 27, 707-715.

Bahramali, H., Gordon, E., Lim, C.L., Li, W., Lagopoulos, J., Leslie, L., Rennie, C., \& Meares, R.A. (1997). Evoked related potentials associated with and without an orienting reflex. Neuroreport, 8, 2665-2669.

Barrett, G., Neshige, R., \& Shibasaki, H. (1987). Human auditory and somatosensory eventrelated potentials, effects of response condition and age. Electroencephalography and Clinical Neurophysiology, 66, 409-437.

\section{Post-print (final draft post-refereeing)}


Bastuji, H.,\&García-Larrea, L. (1999). EPs as a tool for the study of human sleep. Sleep Medicine Reviews, 3, 23-45.

Briem, V., \& Hedman, L.R. (1995). Behavioral effects of mobile telephone use during simulated driving. Ergonomics, 38, 2536-2562.

Brookhuis, K.A.,DeVries, G.,\&DeWaard, D. (1991). The effects of mobile telephoning on driving performance. Accident Analysis and Prevention, 26, 309-316.

Brown, I.D.,Tickner, A.H.,\&Simmons, D.C. (1969). Interference between concurrent tasks of driving and telephoning. Journal of Applied Psychology, 53, 419-424.

Brunia, C.H.M. (1993). Waiting in readiness: Gating in attention and motor preparation. Psychophysiology, 30, 327-339.

Donchin, E. (1979). Event-related potentials: A tool in the study of human information processing. In H. Begleiter (Ed.), Evoked potentials and behavior (pp. 13-88). New York: Plenum.

Eulitz, C., Ullsperger, P., Freude, G., \& Elbert, T. (1998). Mobile phones modulate response patterns of human brain activity. Neuroreport, 9, 3229-3232.

Falkenstein, M., Hohnsbein, J.,\&Hoormann, J. (1994). Effects of choice complexity on different subcomponents of the late positive complex of the event-related potential. Electroencephalography and Clinical Neurophysiology, 92, 148-160.

Freude, G., \& Ullsperger, P. (2000). Do microwaves emitted by cellular phones influence the bioelectrical brain activity? Journal of Psychophysiology, 14, 58 (abstract).

García-Larrea, L.,\&Cézanne-Bert, G. (1998). P3, SlowWave and working memory load. A study on the functional correlates of Slow Wave activity. Electroencephalography and Clinical Neurophysiology, 108, 260-273.

Goodin, D., Desmedt, J.E., Maurer, K., \& Nuwer, M. (1994). IFCN recommended standards for long-latency auditory eventrelated potentials. Report of an IFCN committee. Electroencephalography and Clinical Neurophysiology, 91, 18-20.

Hackley, S.A., \& Valle-Inclán, F.(1998). Automatic alerting does not speed late motoric processes in a reaction-time task. Nature, 391, 786-788.

Isreal, J. B., Chesney, G.B.,Wickens, C.D.,\&Donchin, E. (1980). P300 and tracking difficulty: Evidence for multiple resources in dual-task performance. Psychophysiology, 17, 259273.

Koivisto, M., Revonsuo, A., Krause, C., Haarala, C., Sillanmaki, L., Laine, M., \& Hamalainen, H. (2000). Effects of $902 \mathrm{MHz}$ electromagnetic field emitted by cellular telephones on response times in humans. Neuroreport, 11, 413-415.

\section{Post-print (final draft post-refereeing)}


Kok, A. (1997). Event-related potential (ERP) reflections of mental resources: A review and synthesis. Biological Psychology, 45, 19-56.

Kornhuber, H.H., \& Deecke, L. (1965). Hirnpotentialänderungen bei Willkürbewegungen und passiven Bewegungen des Menschen: Bereitschaftspotential und reafferente Potentiale. Pfluggers Archives, 284, 1-17.

Kutas, M., McCarthy, G., \& Donchin, E. (1977). Augmenting mental chronometry: The P300 as a measure of stimulus evaluation time. Science, 197, 792-795.

Lamble, D., Kauranen, T., Laakso, M., \& Summala, H.(1999). Cognitive load and detection thresholds in car following situations: safety implications for using mobile (cellular) telephones

while driving. Accident Analysis and Prevention, 31, 617-623.

McCallum, W.C. (1988). Potentials related to expectancy, preparation and motor activity. In T.W. Picton (Ed.), Handbook of electroencephalography and clinical neurophysiology (revised series). Vol. 3: Human event-related potentials (pp. 427-533). Amsterdam: Elsevier.

Picton, T.W. (1992). The P300 wave of the event-related potential. Journal of Clinical Neurophysiology, 9, 456-479.

Preece, A.W., Iwi, G., Davies-Smith, A., Wesnes, K., Butler, S., Lim, E., \& Varey, A. (1999). Effect of a $915-\mathrm{MHz}$ simulated mobile phone signal on cognitive function in man. International Journal of Radiation Biology, 75, 447-456.

Ragazzoni, A., Mata, S., Grippo, A., \& Pinto, F. (1996). Dual taskperformance: Effects of increasing difficulty on aufitory ERPs and RTs. In C. Barber, G. Celesia, C. Comi, \& F. Mauguière (Eds.), Functional neuroscience (EEG Suppl. 46, pp. 253-260). Amsterdam: Elsevier.

Ritter W., Simson, R., Vaughan, H.G., \& Friedman, D.A. (1979).A brain event related to the making of sensory discrimination. Science, 203, 1358-1361.

Schubert,M., Johannes, S., Koch, M., Wieringa, B.M., Dengler, R., \& Munte, T.F. (1998). Differential effects of two motor tasks on ERPs in an auditory classification task: Evidence of shared cognitive resources. Neuroscience Research, 30, 125-134.

Sirevaag, E.J., Kramer, A.F., Coles, M.G., \& Donchin, E. (1989). Resource reciprocity: An event related brain potentials analysis. Acta Psychologica, 70, 77-97.

Starr, A., Sandroni, P., \& Michalewski, H.J. (1995). Readiness to respond in a target detection task: Pre- and poststimulus eventrelated potentials in normal subjects. Electroencephalography and Clinical Neurophysiology, 96, 76-92.

Verleger, $R$. (1997). On the utility of $P 3$ latency as an index of mental chronometry. Psychophysiology, 34, 131-156.

\section{Post-print (final draft post-refereeing)}


Walter,W.G., Cooper, R., Aldridge,V.J., McCallum,W.C.,\&Winter, A.L. (1964). Contingent negative variation:An electric sign of sensorimotor association and expectancy in the human

brain. Nature, 203, 380-384.

Wickens, C.D., Kramer, A.F., \& Donchin, E. (1984). The eventrelated potential as an index of the processing demands of a complex target acquisition task. Annals of the New York Academy of Science, 425, 295-299

\section{Post-print (final draft post-refereeing)}


Filter: $0.1-30 \mathrm{~Hz}$

Stim. onset

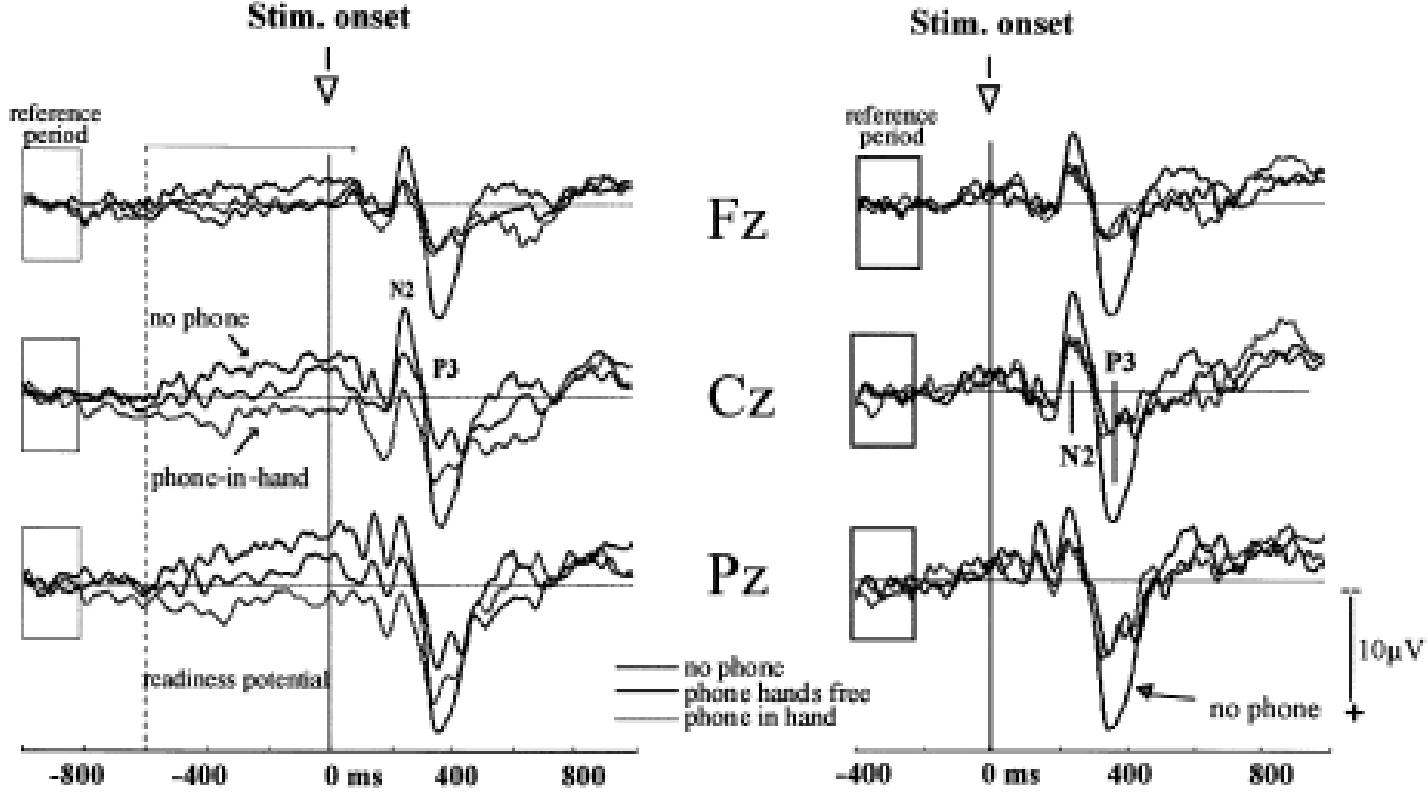

Figure 1 Event-related potentials preceding and following the target stimulus in the three experimental conditions. In the left ponel, the pre-stimulus traces show a slaw negative wave mainly attributed to a "readiness potential" (RP), which develaps during the 500 ms preceding stimulus anset. The RP was maximal in the "no-phone" condition and minimal in the "phone-in-hand" situation, with intermed iatevalyes during the "hands-free" converation. The right pand shows the same traces after high-poss filtering at $1 \mathrm{~Hz}$ performed to eliminate the slow negative drift imposed by the AP. The amplitudes of the N2 and P3 waves were reduced to a similar degree in the "phone-in-hand" and the "hands-free" conditions, relative to the "no-phone" situation. Negativity is up. 


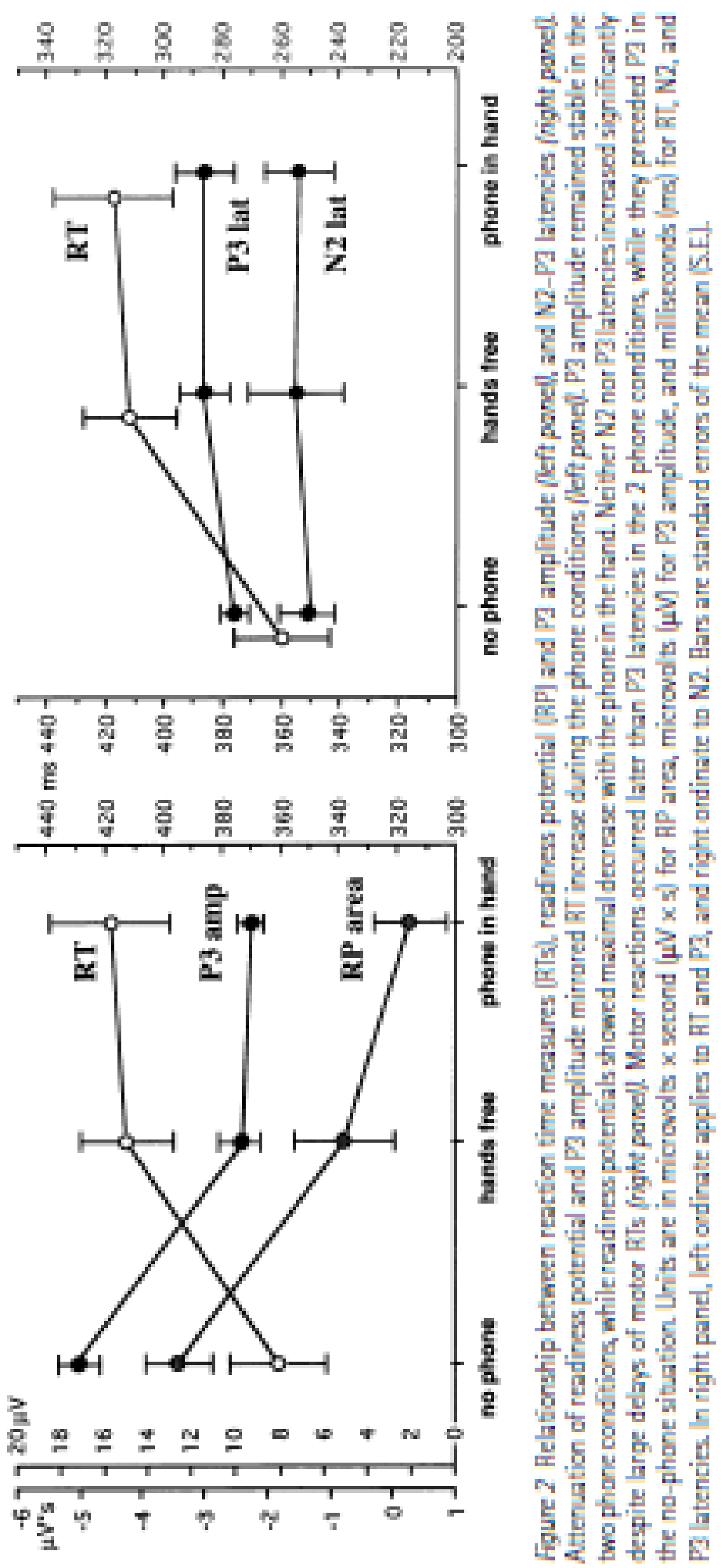

э) 